Mathematical Modelling AND ANALysis

Volume 18 Number 4, September 2013, 489-504

http://dx.doi.org/10.3846/13926292.2013.840686

(c) Vilnius Gediminas Technical University, 2013
Publisher: Taylor\&Francis and VGTU

http://www.tandfonline.com/TMMA

Print ISSN: 1392-6292

Online ISSN: 1648-3510

\title{
Analysis of Global Asymptotic Stability and Pseudo Almost Periodic Solution of a Class of Chaotic Neural Networks
}

\author{
Farouk Chérif ${ }^{a, b}$ \\ ${ }^{a}$ University of Sousse \\ Cité Taffala (Ibn Khaldoun), 4003 Sousse, Tunisia \\ ${ }^{b}$ Ecole Supérieure des Sciences et de Technologie \\ Avenue Lamine Abassi, 4011 Hammam Sousse, Tunisia \\ E-mail: faroukcheriff@yahoo.fr
}

Received August 8, 2012; revised August 22, 2013; published online September 1, 2013

\begin{abstract}
In this paper we give sufficient conditions ensuring the existence and uniqueness of pseudo almost periodic solution of a class of delayed chaotic neural networks. Further, we study the global asymptotic stability (GAS) of the considered model and give a set of criteria on (GAS) by constructing new Lyapunov functional.

Keywords: almost periodic functions, mathematical model, neural networks, contraction mapping principle, delay.
\end{abstract}

AMS Subject Classification: 34K14; 92B20; 93D20.

\section{Introduction}

Pseudo Almost periodic functions are part of a hierarchy of functions which starts with the well known periodic functions and these functions were first introduced in the literature by Zhang [30] as a natural generalization of the class of almost periodic functions (in the sens of $\mathrm{H}$. Bohr). In addition, after considering longterm dynamical behaviour, it has been found that the investigation of almost periodic and pseudo almost periodic behaviour are in more accordance with reality since there is no phenomenon which is purely periodic. Hence, in the past two decades, some works dealing with the existence of almost periodic and/or pseudo almost periodic solutions of nonlinear differential equations have appeared with applications in different fields such as mathematical biology, control and physics $([1,6,7,12,13,15,18,22]$ and the references therein).

As we all know, the chaotic neural networks (CNNs) are non linear dynamic systems characterized by a striking resemblance to the biological neural networks in the human brain. In particular, the chaotic neural network shows the more complex spatial-temporal chaotic dynamics compared to the coupled 
map lattice system in which each lattice site of coupled map lattice systems is only coupled to its nearest ones.

Hence, one of the most important behaviours of solutions which has been a main object of our investigations in previous papers is the almost automorphic and pseudo almost periodic behaviour of solutions of delayed systems such as RNNs, HNNs and SICNNs $[4,9,10,11]$. Besides, it is natural to study some models with delays since those delays are inevitable in the neural networks (see, for example, $[5,22,25]$ and the references therein).

The main motivation of this paper is to study the existence, uniqueness and global asymptotic stability of pseudo almost-periodic solution for a class of chaotic neural network with discrete and distributed delays. Our approach is based mainly on the contraction mapping principle and applying the Brouwer's fixed point Theorem. To the best of author's observation, no work has been published in the literature regarding the oscillatory behaviour for the delayed chaotic neural network.

This paper is organized as follows. In Section 2, we present the basic notations, definitions and properties which are used throughout this work to obtain our results. In particular, the space of pseudo almost periodic functions is defined and some elementary properties are given. Section 4, is devoted to an existence result. Before that, in Section 3, we shall introduce the model and precise some assumptions. The global asymptotic stability will be the subject of Section 5. In Section 6, an illustrative example is given to show the effectiveness of the results obtained. Finally, we draw conclusions.

\section{Preliminaries: The Function Spaces}

Throughout this paper, we will use the following concepts and notations. For a vector $v=\left(v_{1}, \ldots, v_{n}\right)^{T} \in \mathbb{R}^{n}$, let $\|v\|=\left(\sum_{i=1}^{n} v_{i}^{2}\right)^{\frac{1}{2}}$ denote the Euclidean vector norm, and for a matrix $M \in \mathbb{R}^{n \times n}$, let $\|M\|_{2}=\left(\lambda_{\max }\left(M^{T} M\right)\right)^{\frac{1}{2}}$, where $\lambda_{\max }(\cdot)$ represents the largest eigenvalue of a matrix and $T$ denotes the transpose of a matrix. $B C\left(\mathbb{R}, \mathbb{R}^{n}\right)$ denotes the set of bounded continued functions from $\mathbb{R}$ to $\mathbb{R}^{n}$. Note that $\left(B C\left(\mathbb{R}, \mathbb{R}^{n}\right),\|\cdot\|_{\infty}\right)$ is a Banach space where $\|\cdot\|_{\infty}$ denotes the sup norm

$$
\|f\|_{\infty}:=\sup _{t \in \mathbb{R}}\|f(t)\| .
$$

Denote by $A P\left(\mathbb{R}, \mathbb{R}^{n}\right)$ the set of the Bohr-almost periodic functions from $\mathbb{R}$ to $\mathbb{R}^{n}$. One of the definition consists in saying the following:

Definition 1. A continuous function $f \in A P\left(\mathbb{R}, \mathbb{R}^{n}\right)$, if and only if for each $\epsilon>0$, the set

$$
T(f, \epsilon)=\{\tau \in \mathbb{R},|f(t+\tau)-f(\tau)|<\epsilon\}
$$

is relatively dense in $\mathbb{R}$. In other words, there exists $l_{\epsilon}>0$ such that every interval of length $l_{\epsilon}$ contains at least one point of $T(f, \epsilon)$.

The number $\tau$ in Definition 1 is called an $\epsilon$-translation number of the function $f$. We refer the reader to ( $[2]$ and [9]) for the basic theory of almost periodic functions and their applications. 
Define the class of functions $P A P_{0}\left(\mathbb{R}, \mathbb{R}^{n}\right)$ as follows:

$$
\left\{f \in B C\left(\mathbb{R}, \mathbb{R}^{n}\right) / \lim _{T \rightarrow+\infty} \frac{1}{2 T} \int_{-T}^{T}|f(t)| d t=0\right\} .
$$

A function $f \in B C\left(\mathbb{R}, \mathbb{R}^{n}\right)$ is called pseudo almost periodic if it can be expressed as $f=h+\varphi$, where $h \in A P\left(\mathbb{R}, \mathbb{R}^{n}\right)$ and $\varphi \in P A P_{0}\left(\mathbb{R}, \mathbb{R}^{n}\right)$. The collection of such functions will be denoted by $P A P\left(\mathbb{R}, \mathbb{R}^{n}\right)$.

The functions $h$ and $\varphi$ in above definition are respectively called the principal component (almost periodic) and the ergodic perturbation of the pseudo almost periodic function $f$. Furthermore, the decomposition given in definition above is unique.

Remark 1. Notice that $\left(P A P\left(\mathbb{R}, \mathbb{R}^{n}\right)\right.$ is a closed subspace of $B C\left(\mathbb{R}, \mathbb{R}^{n}\right)$. So, $P A P\left(\mathbb{R}, \mathbb{R}^{n}\right)$ endowed with the uniform-norm, is a Banach space. On the other hand, $\left.A P\left(\mathbb{R}, \mathbb{R}^{n}\right)\right)$ is a proper subspace of $P A P\left(\mathbb{R}, \mathbb{R}^{n}\right)$. Indeed, for instance, the function $\phi(t)=\sin ^{2} \pi t+\sin ^{2} t+e^{-t^{t} \cos ^{2} t}$ is pseudo almost periodic function but not almost periodic [11].

\section{Description System}

In this paper, we are concerned with the following delayed chaotic neural networks

$$
\left\{\begin{aligned}
\frac{d x_{i}(t)}{d t}= & -d_{i} x_{i}(t)+\sum_{j=1}^{n} a_{i j} f_{j}\left(x_{j}(t)\right)+\sum_{j=1}^{n} b_{i j} g_{j}\left(x_{j}(t-\tau)\right) \\
& +\int_{t-\sigma}^{t} \sum_{j=1}^{n} c_{i j} h_{j}\left(x_{j}(\rho)\right) d \rho+J_{i}(t),
\end{aligned}\right.
$$

where $n$ is the number of the neurons in the neural network, $x_{i}(t)$ denotes the state of the $i$ th neural neuron at time $t, f_{j}\left(x_{j}(t)\right), g_{j}\left(x_{j}(t)\right)$ and $h_{j}\left(x_{j}(t)\right)$ are the activation functions of $j$ th neuron at time $t$. The constants $a_{i j}, b_{i j}$ and $c_{i j}$ denote, respectively, the connection weights, the discretely delayed connection weights, and the distributively delayed connection weights, of the $j$ th neuron on the $i$ neuron, $J_{i}(\cdot)$ is the external bias on the $i$ th neuron, $d_{i}$ denotes the rate with which the $i$ th neuron will reset its potential to the resting state in isolation when disconnected from the network and external inputs. $\tau$ is the constant discrete time delay, while $\sigma$ describes the distributed time delay. The initial condition associated with (3.1) is given in the form

$$
x_{i}(t)=\varphi_{i}(t) \text { for } i \in\{1,2, \ldots, n\} \text { and } t \in[-\alpha, 0] \text {, }
$$

where for all $i \in\{1,2, \ldots, n\}$ the numerical function $\varphi_{i}(\cdot)$ is continuous on $[-\alpha, 0]$ with $\alpha=\max (\tau, \sigma)$. We denote a vector solution of the above system as

$$
x(t)=\left(x_{1}(t), x_{2}(t), \ldots, x_{n}(t)\right)^{T},
$$


where $T$ denotes the transpose of a matrix and

$$
\begin{cases}x_{i}(t)=x_{i}\left(t, \varphi_{i}\right), & \text { if } t \geq 0 \\ x_{i}(t)=\varphi_{i}(t), & \text { if }-\alpha \leq t \leq 0\end{cases}
$$

The neural network (3.1) can be rewritten in the following matrix-vector form

$$
\left\{\begin{aligned}
\dot{x}(t)= & -D x(t)+A f(x(t))+B g(x(t-\tau))) & & \\
& +C \int_{t-\sigma}^{t} h(x(\rho)) d \rho+J(t), & & t \geq 0, \\
x(t)= & \varphi(t), & & -\alpha \leq t \leq 0 .
\end{aligned}\right.
$$

Throughout this paper, we consider the following assumptions:

$\left(H_{1}\right)$ The activity functions $f, g, h$ are assumed to be global Lipschitz continuous, that is, there exist $L_{f}, L_{g}, L_{h}>0$ such that for all $x, y \in \mathbb{R}^{n}$

$$
\begin{aligned}
& \|f(x)-f(y)\|<L_{f}\|x-y\|, \quad\|g(x)-g(y)\|<L_{g}\|x-y\|, \\
& \|h(x)-h(y)\|<L_{h}\|x-y\| .
\end{aligned}
$$

Furthermore, we suppose that $f(0)=g(0)=h(0)=0$.

$\left(H_{2}\right) \quad J(\cdot) \in P A P\left(\mathbb{R}, \mathbb{R}^{n}\right)$ and $\tau, \sigma>0$.

$\left(H_{3}\right) \quad r=\frac{1}{d}\left(L_{f}\|A\|_{2}+L_{g}\|B\|_{2}+\sigma L_{h}\|C\|_{2}\right)<1$, where $d=\min _{1 \leq i \leq n} d_{i}$.

$\left(H_{4}\right) \quad d>\sum_{j=1}^{n}\left(\left|a_{i j}\right| L_{f}+\left|b_{i j}\right| L_{g}+\sigma\left|c_{i j}\right| L_{h}\right)$.

\section{Existence and Uniqueness of Pseudo Almost Periodic Solution}

In this section, we establish some results for the existence, uniqueness of pseudo almost periodic solution of (3.2). This setting requires the following preliminary and technical lemmas.

Lemma 1. [11] For all $\beta \in \mathbb{R}, x(\cdot-\beta) \in P A P\left(\mathbb{R}, \mathbb{R}^{n}\right)$ whenever $x(\cdot) \in$ $P A P\left(\mathbb{R}, \mathbb{R}^{n}\right)$.

Lemma 2. Suppose that assumption $\left(H_{1}\right)$ holds and $x(\cdot) \in P A P\left(\mathbb{R}, \mathbb{R}^{n}\right)$ then $\phi: t \longmapsto \int_{t-\sigma}^{t} h(x(\rho)) d \rho$ belongs to $\operatorname{PAP}\left(\mathbb{R}, \mathbb{R}^{n}\right)$.

Proof. By the composition Theorem of pseudo-almost periodic functions [3], the function $\psi: t \mapsto h(x(t))$ belongs to $P A P\left(\mathbb{R}, \mathbb{R}^{n}\right)$ whenever $x \in P A P\left(\mathbb{R}, \mathbb{R}^{n}\right)$, then $\psi$ can be expressed as $\psi=u+v$, where $u \in A P\left(\mathbb{R}, \mathbb{R}^{n}\right)$ and $v \in$ $P A P_{0}\left(\mathbb{R}, \mathbb{R}^{n}\right)$. Consequently,

$$
\phi(t)=\int_{t-\sigma}^{t} u(\rho) d \rho+\int_{t-\sigma}^{t} v(\rho) d \rho=\phi_{1}(t)+\phi_{2}(t) .
$$


Let us prove the almost periodicity of the function $t \longmapsto \phi_{1}(t)$. For an arbitrary $\varepsilon>0$, we consider, in view of the almost periodicity of the function $u$, a number $L_{\varepsilon}$ such that in any interval $[\alpha, \alpha+L[$ one finds a number $\delta$, with property that:

$$
\sup _{t \in \mathbb{R}}\|u(t+\delta)-u(t)\|<\frac{\varepsilon}{\sigma} .
$$

Afterwards, we can write:

$$
\left\|\phi_{1}(t+\delta)-\phi_{1}(t)\right\| \leq \int_{t-\sigma}^{t}\|u(\xi+\delta)-u(\xi)\| d \xi \leq \varepsilon
$$

which implies that $\phi_{1}(\cdot) \in A P\left(\mathbb{R}, \mathbb{R}^{n}\right)$. Now, we turn our attention to $\phi_{2}(\cdot)$. First, note that $s \longmapsto \phi_{2}(s)$ is a bounded continuous function. Thus, it remains to prove that

$$
\lim _{T \rightarrow+\infty} \frac{1}{2 T} \int_{-T}^{T}\left\|\phi_{2}(s)\right\| d s=0 .
$$

Let us take any $\epsilon>0$ and set $C_{\epsilon}:=\{t \in \mathbb{R} ;\|v(t)\| \geq \epsilon\}$. According to [19]

$$
\frac{m\left(C_{\varepsilon} \cap[-T, T]\right)}{2 T} \longrightarrow 0
$$

as $T \longrightarrow+\infty$, where $m$ is the Lebesgue measure. So

$$
\begin{aligned}
\frac{1}{2 T} \int_{-T}^{T}\left\|\int_{t-\sigma}^{t} v(\rho) d \rho\right\| d t & \leq \frac{1}{2 T} \int_{-T}^{T}\left(\int_{t-\sigma}^{t}\|v(\rho)\| d \rho\right) d t \\
& =\frac{1}{2 T} \int_{[-T, T] \cap C \varepsilon}+\int_{[-T, T]-C \varepsilon}\left(\int_{t-\sigma}^{t}\|v(\rho)\| d \rho\right) d t \\
& \leq\|v\| \sigma\left(\frac{m\left(C_{\varepsilon} \cap[-T, T]\right)}{2 T}\right)+\varepsilon \sigma \longrightarrow 0
\end{aligned}
$$

as $T \longrightarrow+\infty$. The proof of Lemma 2 is now complete.

Lemma 3. Suppose that assumptions $\left(H_{1}\right),\left(H_{2}\right)$ hold. Define the nonlinear operator $\Gamma$ by: for each $\varphi \in P A P\left(\mathbb{R}, \mathbb{R}^{n}\right)$

$(\Gamma \varphi)(t)=\int_{-\infty}^{t} e^{-(t-s) D}\left[A f(\varphi(s))+B g(\varphi(s-\tau))+C \int_{s-\sigma}^{s} h(\varphi(\rho)) d \rho+J(s)\right] d s$.

Then $\Gamma$ maps $P A P\left(\mathbb{R}, \mathbb{R}^{n}\right)$ into itself.

Proof. First, let us check that $\Gamma$ is well defined. Indeed, by Lemma 1, for all if $\varphi \in P A P\left(\mathbb{R}, \mathbb{R}^{n}\right)$ the function $T_{h}(\varphi)=\varphi(\cdot-h) \in P A P\left(\mathbb{R}, \mathbb{R}^{n}\right)$. And hence, by the composition Theorem of pseudo-almost periodic functions [3], the functions $t \longmapsto A f(\varphi(t))$ and $t \longmapsto B g(\varphi(t-\tau))$ belong to $P A P\left(\mathbb{R}, \mathbb{R}^{n}\right)$ whenever $\varphi \in P A P\left(\mathbb{R}, \mathbb{R}^{n}\right)$. Thus,

$$
t \longmapsto F(t)=A f(\varphi(t))+B g(\varphi(t-\tau))+C \int_{t-\sigma}^{t} h(\varphi(\rho)) d \rho+J(t)
$$


belongs to $P A P\left(\mathbb{R}, \mathbb{R}^{n}\right)$. Consequently the integral (in Riemann's sense)

$$
\int_{R}^{t} e^{-(t-s) D}\left[A f(\varphi(s))+B g(\varphi(s-\tau))+C \int_{s-\sigma}^{s} h(\varphi(\rho)) d \rho+J(s)\right] d s
$$

has a sense. Besides, the integrand is estimated by $M\left\|e^{-(t-s) D}\right\|$ where

$$
M=\sup _{s \in \mathbb{R}}\left\|A f(\varphi(s))+B g(\varphi(s-\tau))+C \int_{s-\sigma}^{s} h(\varphi(\rho)) d \rho+J(s)\right\|<+\infty .
$$

Thus, the integral

$$
\int_{-\infty}^{t} e^{-(t-s) D}\left[A f(\varphi(s))+B g(\varphi(s-\tau))+C \int_{s-\sigma}^{s} h(\varphi(\rho)) d \rho+J(s)\right] d s
$$

is absolutely convergent and

$$
\|(\Gamma \varphi)(t)\| \leq M \int_{-\infty}^{t}\left\|e^{-(t-s) D}\right\| d s \leq M \int_{-\infty}^{t} e^{-(t-s) d} d s=\frac{M}{d} .
$$

Since the function $F$ belongs to $P A P\left(\mathbb{R}, \mathbb{R}^{n}\right)$, then $F$ can be expressed as

$$
F=F_{1}+F_{2}
$$

where $F_{1} \in A P\left(\mathbb{R}, \mathbb{R}^{n}\right)$ and $F_{2} \in P A P_{0}\left(\mathbb{R}, \mathbb{R}^{n}\right)$. Consequently,

$$
(\Gamma F)=\left(\Gamma F_{1}\right)+\left(\Gamma F_{2}\right),
$$

where

$$
\left(\Gamma F_{1}\right)(t)=\int_{-\infty}^{t} e^{-(t-s) D} F_{1}(s) d s \quad \text { and } \quad\left(\Gamma F_{2}\right)(t)=\int_{-\infty}^{t} e^{-(t-s) D} F_{2}(s) d s .
$$

Let us prove the almost periodicity of $t \longmapsto\left(\Gamma F_{1}\right)(t)$. For $\varepsilon>0$, we consider, in view of the almost periodicity of $F_{1}$, a number $L(\varepsilon, d)$ such that in any interval $[\mu, \mu+L[$ one finds a number $\delta$, with property that:

$$
\sup _{t \in \mathbb{R}}\left\|F_{1}(t+\delta)-F_{1}(t)\right\|<\varepsilon d .
$$

Directly afterwards, we can write

$$
\left\|\left(\Gamma F_{1}\right)(t+\tau)-\left(\Gamma F_{1}\right)(t)\right\| \leq \int_{-\infty}^{t}\left\|e^{-(t-\xi) D}\right\|\left\|F_{1}(\xi+\tau)-F_{1}(\xi)\right\| d \xi \leq \varepsilon,
$$

which implies the almost periodicity of $\left(\Gamma F_{1}\right)$. Now, we turn our attention to $\left(\Gamma F_{2}\right)$. First, note that $s \longmapsto\left(\Gamma F_{2}\right)(s)$ is a bounded continuous function. Thus, it remains to prove that

$$
\lim _{T \rightarrow+\infty} \frac{1}{2 T} \int_{-T}^{T}\left\|\left(\Gamma F_{2}\right)(s)\right\| d s=0 .
$$


Clearly,

$$
\lim _{T \rightarrow+\infty} \frac{1}{2 T} \int_{-T}^{T}\left\|\left(\Gamma F_{2}\right)(s)\right\| d s \leq I+K
$$

where

$$
I=\lim _{T \rightarrow+\infty} \frac{1}{2 T} \int_{-T}^{T} d t\left(\int_{-T}^{t}\left\|e^{-(t-s) D} F_{2}(s)\right\| d s\right)
$$

and

$$
K=\lim _{T \rightarrow+\infty} \frac{1}{2 T} \int_{-T}^{T} d t\left(\int_{-\infty}^{-T}\left\|e^{-(t-s) D} F_{2}(s)\right\| d s\right) .
$$

It is clear that

$$
\begin{aligned}
I & =\lim _{T \rightarrow+\infty} \frac{1}{2 T} \int_{-T}^{T} d t\left(\int_{-T}^{t}\left\|e^{-(t-s) D} F_{2}(s)\right\| d s\right) \\
& \leq \lim _{T \rightarrow+\infty} \frac{1}{2 T d} \int_{-T}^{T}\left\|F_{2}(t)\right\| d t=0 .
\end{aligned}
$$

Reasoning in a similar way we obtain the following estimates

$$
\begin{aligned}
K & =\lim _{T \rightarrow+\infty} \frac{1}{2 T} \int_{-T}^{T} d t\left(\int_{-\infty}^{-T}\left\|e^{-(t-s) D} F_{2}(s)\right\| d s\right) \\
& \leq \lim _{T \rightarrow+\infty} \frac{\sup _{t \in \mathbb{R}}\left\|F_{2}(t)\right\|}{d} e^{-2 d T}=0 .
\end{aligned}
$$

Consequently, the function $\left(\Gamma F_{2}\right)$ belongs to $P A P_{0}\left(\mathbb{R}, \mathbb{R}^{n}\right)$, and hence $(\Gamma F)=$ $\left(\Gamma F_{1}\right)+\left(\Gamma F_{2}\right) \in P A P\left(\mathbb{R}, \mathbb{R}^{n}\right)$. The proof is complete.

Theorem 1. Suppose that assumptions $\left(H_{1}\right)-\left(H_{3}\right)$ hold. Then the delayed chaotic neural networks (3.1) has a unique pseudo almost periodic solution in the convex

$$
\mathcal{B}=B\left(\varphi_{0}, R\right)=\left\{\varphi \in P A P\left(\mathbb{R}, \mathbb{R}^{n}\right),\left\|\varphi-\varphi_{0}\right\| \leq \frac{r\|J\|_{\infty}}{d(1-r)}\right\}
$$

where

$$
\varphi_{0}(t)=\int_{-\infty}^{t} e^{-(t-s) D} J(s) d s
$$

Proof. Clearly, $\mathcal{B}$ is a closed convex subset of $P A P\left(\mathbb{R}, \mathbb{R}^{n}\right)$ and

$$
\left\|\varphi_{0}(t)\right\|=\|J\|_{\infty} / d
$$

Therefore, for any $\varphi \in \mathcal{B}$ by using the estimate just obtained, we see that

$$
\|\varphi\| \leq\left\|\varphi-\varphi_{0}\right\|+\left\|\varphi_{0}\right\| \leq \frac{r\|J\|_{\infty}}{d(1-r)}+\frac{\|J\|_{\infty}}{d}=\frac{\|J\|_{\infty}}{d(1-r)} .
$$

It is clear that the operator $\Gamma$ is a self-mapping from $\mathcal{B}$ to $\mathcal{B}$. In fact, for any $\varphi \in \mathcal{B}$, we have

$$
\left\|(\Gamma \varphi)(t)-\varphi_{0}(t)\right\| \leq \frac{L_{f}\|A\|_{2}+L_{g}\|B\|_{2}+\sigma L_{h}\|C\|_{2}}{d}\|\varphi\|_{\infty} \leq \frac{r\|J\|_{\infty}}{d(1-r)},
$$


which implies that $(\Gamma \varphi) \in \mathcal{B}$. Next, we prove that the mapping $\Gamma$ is a contraction mapping of $\mathcal{B}$. In view of $\left(H_{2}\right)$, for any $\varphi, \psi \in \mathcal{B}$, we have

$$
\begin{aligned}
& \|(\Gamma \varphi)(t)-(\Gamma \psi)(t)\| \leq \int_{-\infty}^{t} e^{-(t-s) d}\|A[f(\varphi(s))-f(\psi(s))]\| d s \\
& \quad+\int_{-\infty}^{t} e^{-(t-s) d}\|B[g(\varphi(s-\tau))-g(\psi(s-\tau))]\| d s \\
& \quad+\int_{-\infty}^{t}\left\|C \int_{s-\sigma}^{s}[h(\varphi(\rho))-h(\psi(\rho))] d \rho\right\| d s \\
& \leq \frac{1}{d}\left(L_{f}\|A\|_{2}+L_{g}\|B\|_{2}+\sigma L_{h}\|C\|_{2}\right)\|\varphi-\psi\|_{\infty} \leq r\|\varphi-\psi\|_{\infty} .
\end{aligned}
$$

The assumption $\left(H_{3}\right)$ implies that $\Gamma$ is a contraction mapping on $\mathcal{B}$. Then there exists a unique fixed point $\varphi_{*} \in \mathcal{B}$ such that $\Gamma \varphi_{*}=\varphi_{*}$. This implies that (3.1) has a unique pseudo almost periodic solution in $\mathcal{B} \subset P A P\left(\mathbb{R}, \mathbb{R}^{n}\right)$.

\section{Global Asymptotic Stability}

In this section we will assume that the impulsive function is constant. Let us consider the special case of the model (3.1)

$$
\left\{\begin{array}{rl}
\frac{d x_{i}(t)}{d t}= & -d_{i} x_{i}(t)+\sum_{j=1}^{n} a_{i j} f_{j}\left(x_{j}(t)\right) \\
& +\sum_{j=1}^{n} b_{i j} g_{j}\left(x_{j}(t-\tau)\right)+\int_{t-\sigma}^{t} \sum_{j=1}^{n} c_{i j} h_{j}\left(x_{j}(\rho)\right) d \rho+J_{i}
\end{array} .\right.
$$

Accompanying the network above by an initial condition of the form

$$
\left\{\begin{array}{ll}
x_{i}(t)=x_{i}\left(t, \varphi_{i}\right), & \text { if } t \geq 0 \\
x_{i}(t)=\varphi_{i}(t), & \text { if }-\alpha \leq t \leq 0
\end{array} \quad \text { for all } i \in\{1,2, \ldots, n\} .\right.
$$

We denote a vector solution of the above system as

$$
x(t)=\left(x_{1}(t), x_{2}(t), \ldots, x_{n}(t)\right)^{T} .
$$

Hence, the neural network (5.1) can be rewritten in the following matrix-vector form

$$
\left\{\begin{aligned}
\dot{x}(t)= & -D x(t)+A f(x(t))+B g(x(t-\tau)) & & \\
& +C \int_{t-\sigma}^{t} h(x(\rho)) d \rho+J, & & t \geq 0, \\
x(t)= & \varphi(t), & & -\alpha \leq t \leq 0 .
\end{aligned}\right.
$$

Definition 2. [17] Let $f: \mathbb{R} \longrightarrow \mathbb{R}$ be a continuous function, then $\frac{D^{+} f(t)}{d t}$ is defined as

$$
\frac{D^{+} f(t)}{d t}=\varlimsup_{h \rightarrow 0^{+}} \frac{f(t+h)-f(t)}{h} .
$$


Remark 2. The upper-right Dini derivative $\frac{D^{+} V|f(t)|}{d t}$ of $|f(t)|$ is given by

$$
\frac{D^{+} V|f(t)|}{d t}=\operatorname{sign}(f(t)) \frac{d f(t)}{d t},
$$

where $\operatorname{sign}(\cdot)$ is the signum function.

Definition 3. A constant vector $x^{*}=\left(x_{1}^{*}, \ldots, x_{n}^{*}\right)^{T}$ is said to be an equilibrium solution to the above equation if it satisfies the system of equations

$$
-d_{i} x_{i}^{*}+\sum_{j=1}^{n} a_{i j} f_{j}\left(x_{j}^{*}\right)+\sum_{j=1}^{n} b_{i j} g_{j}\left(x_{j}^{*}\right)+\sigma \sum_{j=1}^{n} c_{i j} h_{j}\left(x_{j}^{*}\right)+J_{i}=0
$$

for $1 \leq i \leq n$.

Theorem 2. Let the conditions $\left(H_{1}\right)-\left(H_{4}\right)$ hold. Then the equation (5.1) possess an equilibrium point.

Proof. First, let us prove the existence and uniqueness of the equilibrium $x^{*}$ of the equation (5.1). Consider a mapping

$$
\theta\left(x_{1}, x_{2}, \ldots, x_{n}\right)=\left(\begin{array}{c}
\theta_{1}\left(x_{1}, x_{2}, \ldots, x_{n}\right) \\
\theta_{2}\left(x_{1}, x_{2}, \ldots, x_{n}\right) \\
\vdots \\
\theta_{n}\left(x_{1}, x_{2}, \ldots, x_{n}\right)
\end{array}\right)
$$

where for all $i \in\{1, \ldots, n\}$

$$
x_{i}=\theta_{i}\left(x_{1}, x_{2}, \ldots, x_{n}\right)=\frac{1}{d_{i}}\left\{\sum_{j=1}^{n}\left(a_{i j} f_{j}\left(x_{j}\right)+b_{i j} g_{j}\left(x_{j}\right)+\sigma c_{i j} h_{j}\left(x_{j}\right)\right)+J_{i}\right\} .
$$

It is clear that for all $i \in\{1, \ldots, n\}$

$$
\left|\theta_{i}\left(x_{1}, \ldots, x_{n}\right)\right| \leq \frac{1}{d_{i}}\left\{\sum_{j=1}^{n}\left(\left|a_{i j}\right| M_{f}+\left|b_{i j}\right| M_{g}+\sigma M_{h}\left|c_{i j}\right|+\left|J_{i}\right|\right)\right\}=\omega .
$$

One can see without difficulty that

$$
x=\left(x_{1}, x_{2}, \ldots, x_{n}\right)^{T} \in[-\omega, \omega]^{n} \Longrightarrow \theta\left(x_{1}, x_{2}, \ldots, x_{n}\right) \in[-\omega, \omega]^{n} .
$$

Besides, the function

$$
\theta:[-\omega, \omega]^{n} \longrightarrow[-\omega, \omega]^{n}
$$

is continuous since for all $i \in\{1,2, \ldots, n\}$ the functions $f_{j}(\cdot), g_{j}(\cdot)$ and $h_{j}(\cdot)$ are continuous. So, by Brouwer's fixed point Theorem [26], there exists at least one fixed point $x^{*} \in[-\omega, \omega]^{n}$ of $\theta$, namely

$$
x_{i}^{*}=\theta_{i}\left(x_{1}^{*}, x_{2}^{*}, \ldots, x_{n}^{*}\right)=\frac{1}{d_{i}}\left\{\sum_{j=1}^{n}\left(a_{i j} f_{j}\left(x_{j}^{*}\right)+b_{i j} g_{j}\left(x_{j}^{*}\right)+\sigma c_{i j} h_{j}\left(x_{j}^{*}\right)\right)+J_{i}\right\},
$$


which proves that $x^{*}$ is an equilibrium point of the equation (4). In the following we will show the uniqueness of the equilibrium point $x^{*}$ of the map $\theta$. For this, we will use the contradiction method. Suppose there exists another fixed point denoted by $y^{*}=\left(y_{1}^{*}, y_{2}^{*}, \ldots, y_{n}^{*}\right)$ such that for all $i \in\{1,2, \ldots, n\}$ one has

$$
y_{i}^{*}=\frac{1}{d_{i}}\left\{\sum_{j=1}^{n}\left(a_{i j} f_{j}\left(y_{j}^{*}\right)+b_{i j} g_{j}\left(y_{j}^{*}\right)+\sigma c_{i j} h_{j}\left(y_{j}^{*}\right)\right)+J_{i}\right\} .
$$

It follows that for all $i \in\{1,2, \ldots, n\}$

$$
\begin{aligned}
d_{i}\left(x_{i}^{*}-y_{i}^{*}\right)= & \sum_{j=1}^{n}\left(a_{i j}\left(f_{j}\left(x_{j}^{*}\right)-f_{j}\left(y_{j}^{*}\right)\right)\right. \\
& \left.+b_{i j}\left(g_{j}\left(x_{j}^{*}\right)-g_{j}\left(y_{j}^{*}\right)\right)+\sigma c_{i j}\left(h_{j}\left(x_{j}^{*}\right)-h_{j}\left(y_{j}^{*}\right)\right)\right) .
\end{aligned}
$$

By $\left(H_{1}\right)$, one has

$$
\begin{aligned}
d_{i}\left|x_{i}^{*}-y_{i}^{*}\right| \leq & \sum_{j=1}^{n}\left(\left|a_{i j}\right| L_{f}\left|x_{j}^{*}-y_{j}^{*}\right|\right. \\
& \left.+\left|b_{i j}\right| L_{g}\left|x_{j}^{*}-y_{j}^{*}\right|+\sigma\left|c_{i j}\right| L_{h}\left|x_{j}^{*}-y_{j}^{*}\right|\right) \\
= & \sum_{j=1}^{n}\left(\left|a_{i j}\right| L_{f}+\left|b_{i j}\right| L_{g}+\sigma\left|c_{i j}\right| L_{h}\right)\left|x_{j}^{*}-y_{j}^{*}\right| .
\end{aligned}
$$

Hence for all $i \in\{1,2, \ldots, n\}$

$$
\sum_{j=1}^{n}\left\{d_{i}-\sum_{j=1}^{n}\left(\left|a_{i j}\right| L_{f}+\left|b_{i j}\right| L_{g}+\sigma\left|c_{i j}\right| L_{g}\right)\right\}\left|x_{i}^{*}-y_{i}^{*}\right| \leq 0 .
$$

By $\left(H_{4}\right)$ it follows that for all $i \in\{1,2, \ldots, n\}, x_{i}^{*}=y_{i}^{*}$, which proves the unicity of the equilibrium point.

Theorem 3. Under the assumptions $\left(H_{1}\right)-\left(H_{4}\right)$ the equilibrium $x^{*}$ of equation (5.1) is globally asymptotically stable independent of delays in the sense that all solutions of (5.1) corresponding to the initial values (5.2) satisfy for all $i \in\{1,2, \ldots, n\}$

$$
\lim _{t \rightarrow+\infty} x_{i}(t)=x_{i}^{*} .
$$

Proof. Let us prove the global exponential stability of the equilibrium $x^{*}$ of the equation (5.1). By using the upper right derivative one has

$$
\begin{aligned}
\frac{d^{+}}{d t}\left|x_{i}(t)-x_{i}^{*}\right| \leq & -d_{i}\left|x_{i}(t)-x_{i}^{*}\right| \\
& +\sum_{j=1}^{n}\left(\left|a_{i j}\right| L_{f}\left|x_{j}(t)-x_{j}^{*}\right|+\left|b_{i j}\right| L_{g}\left|x_{j}(t-\tau)-x_{j}^{*}\right|\right. \\
& \left.+\int_{t-\sigma}^{t} \sum_{j=1}^{n}\left|c_{i j}\right| L_{h}\left|x_{j}(\rho)-x_{j}^{*}\right| d \rho\right) .
\end{aligned}
$$


Pose

$$
\zeta=\min _{1 \leq i \leq n}\left\{d_{i}-\sum_{j=1}^{n}\left(\left|a_{i j}\right| L_{f}+\left|b_{i j}\right| L_{g}+\sigma L_{h}\left|c_{i j}\right|\right)\right\} .
$$

Obviously for all $i \in\{1,2, \ldots, n\}$

$$
d_{i}-\sum_{j=1}^{n}\left(\left|a_{i j}\right| L_{f}+\left|b_{i j}\right| L_{g}+\sigma L_{h}\left|c_{i j}\right|\right) \geq \zeta
$$

Consider for $i \in\{1,2, \ldots, n\}$ the function $\psi_{i}(\cdot)$ defined by

$$
\psi_{i}(t)=t-d_{i}+L_{f} \sum_{j=1}^{n}\left|a_{i j}\right|+L_{g} \sum_{j=1}^{n}\left|b_{i j}\right| e^{t \tau}+\sigma L_{h} \sum_{j=1}^{n}\left|c_{i j}\right| .
$$

It is clear that the functions $t \longmapsto \psi_{i}(t)$ are continuous on $\mathbb{R}^{+}$and by hypothesis $\left(H_{4}\right), \psi_{i}(0)<0$. Thus, there exists a sufficiently small constant $\mu$ such that

$$
\psi_{i}(\mu)<0, \quad \text { for all } 1 \leq i \leq n .
$$

Take an arbitrary $\epsilon>0$. Set, for all $1 \leq i \leq n$,

$$
z_{i}(t)=\left|x_{i}(t)-x_{i}^{*}\right| e^{\mu t}
$$

Then for all $1 \leq i \leq n$, and for all $-\alpha \leq t \leq 0$, one has

$$
z_{i}(t) \leq M<M+\epsilon
$$

where $M=\sup _{-\alpha \leq t \leq 0}\left\|x(t)-x^{*}\right\|$. In the following, we shall prove that for all $t>0$

$$
z_{i}(t) \leq M+\epsilon
$$

Suppose the contrary. Let us denote $A_{i}=\left\{t>0, z_{i}(t)>M+\epsilon\right\}$. It follows that there exists $1 \leq i_{0} \leq n$ such that $A_{i_{0}} \neq \emptyset$. Let

$$
t_{i}= \begin{cases}\inf A_{i}, & \text { if }\left\{t>0, z_{i}(t)>M+\epsilon\right\} \neq \emptyset, \\ +\infty, & \text { if }\left\{t>0, z_{i}(t)>M+\epsilon\right\}=\emptyset .\end{cases}
$$

Then $t_{i}>0$ and for all $-\tau \leq t<t_{i}$, one has

$$
z_{i}(t) \leq M+\epsilon
$$

Let us denote $t_{s}=\min _{1 \leq i \leq n} t_{i}$. It follows that $0<t_{s}<+\infty$ and for all $-\tau \leq t \leq$ $t_{s}$, one has

$$
z_{i}(t) \leq M+\epsilon
$$

Note that

$$
z_{s}\left(t_{s}\right)=M+\epsilon \quad \text { and } \quad D^{+} z_{s}\left(t_{s}\right) \geq 0 .
$$

Now since $x_{i}(\cdot)$ are solutions of Eq. (4), we get

$$
0 \leq D^{+} z_{s}\left(t_{s}\right)=D^{+}\left[\left|x_{s}(t)-x_{i}^{*}\right| e^{\mu t}\right]_{\mid t=t_{s}}
$$




$$
\begin{aligned}
= & \left.\frac{D^{+} z_{i}(t)}{d t}\right|_{t=t_{s}}=e^{\mu t_{s}}\left[\alpha\left|x_{s}(t)-x_{i}^{*}\right|+\left.\frac{D^{+}\left|x_{i}(t)-x_{i}^{*}\right|}{d t}\right|_{t=t_{s}}\right] \\
\leq & \left|x_{s}\left(t_{s}\right)-x_{i}^{*}\right| \mu e^{\mu t_{s}}+e^{\alpha t_{s}}\left(-d_{i}\left|x_{i}\left(t_{s}\right)-x_{i}^{*}\right|+\sum_{j=1}^{n}\left|a_{i j}\right| L_{f}\left|x_{j}\left(t_{s}\right)-x_{j}^{*}\right|\right. \\
& \left.+\left|b_{i j}\right| L_{g}\left|x_{j}\left(t_{s}-\tau\right)-x_{j}^{*}\right|+\int_{t_{s}-\sigma}^{t_{s}} \sum_{j=1}^{n}\left|c_{i j}\right| L_{h}\left|x_{j}(\rho)-x_{j}^{*}\right| d \rho\right) \\
\leq & z_{s}\left(t_{s}\right) \mu e^{\mu t_{s}}+\left(-d_{i} z_{s}\left(t_{s}\right)+\sum_{j=1}^{n}\left|a_{i j}\right| L_{f} z_{s}\left(t_{s}\right)\right. \\
& \left.+\left|b_{i j}\right| L_{g} z_{j}\left(t_{s}-\tau\right) e^{\mu \tau}+\int_{t_{s}-\sigma}^{t_{s}} \sum_{j=1}^{n}\left|c_{i j}\right| L_{h} z_{j}(\rho) e^{-\alpha \rho} d \rho\right) \\
\leq & (M+\epsilon)\left(\left(\mu-d_{s}\right)+\sum_{j=1}^{n}\left(\left|a_{i j}\right| L_{f}+e^{\alpha \tau}\left|b_{i j}\right| L_{g}+\left|c_{i j}\right| L_{h} \int_{t_{s}-\sigma}^{t_{s}} e^{-\alpha \rho} d \rho\right)\right) \\
\leq & (M+\epsilon)\left(\left(\mu-d_{s}\right)+\sum_{j=1}^{n}\left(\left|a_{i j}\right| L_{f}+e^{\mu \tau}\left|b_{i j}\right| L_{g}+\sigma\left|c_{i j}\right| L_{h}\right)\right) .
\end{aligned}
$$

It follows that

$$
\left(\mu-d_{i}\right)+\sum_{j=1}^{n}\left(\left|a_{i j}\right| L_{f}+e^{\mu \tau}\left|b_{i j}\right| L_{g}+\frac{e^{\mu \sigma}-1}{\alpha}\left|c_{i j}\right| L_{h}\right) \geq 0,
$$

that is $\psi_{i}(\mu) \geq 0$, which contradicts the fact that $\psi_{i}(\alpha)<0$. Thus we obtain that for all $t>0$,

$$
z_{i}(t)=\left|x_{i}(t)-x_{i}^{*}\right| \leq(M+\epsilon) e^{-\mu t} .
$$

Note that $\|x(t)-\varphi(t)\|=\max _{1 \leq i \leq n}\left|x_{i}(t)-\varphi_{i}(t)\right|$, then passing to limit when $\epsilon \rightarrow 0^{+}$we obtain for all $t>0$

$$
\left\|x(t)-x^{*}\right\| \leq M e^{-\alpha t}
$$

The proof of this theorem is now completed.

Remark 3. If we let $f=g=h$ equation (3.2) changes into the model of reference [9] where we establish only the existence of the solutions. Hence, this paper is a natural continuation of our previous papers $[9,11]$.

Remark 4. Recently, some sufficient conditions have been obtained for global asymptotic stability (GAS) of delayed neural networks. For instance, the existence and uniqueness of the equilibrium point and its global asymptotic stability are discussed for a class of neural networks with time-varying delays and Lipschitz continuous activation functions by Cao and Wang [8].

Remark 5. When for all $1 \leq i, j \leq, c_{i j}=0$ system (3.1) can be reduced to the model of [28]. Further, our results can be seen as a natural generalization and improvement of [28] since in the model of [28] the authors considered the periodic case. Moreover, this model has attracted a lot of interest especially the study of the problem of synchronization [24,27]. 


\section{An Example}

Let us consider the system

$$
\dot{x}(t)=-D x(t)+A f(x(t))+B f(x(t-\tau))+C \int_{t-\sigma}^{t} f(x(\rho)) d \rho+J(t)
$$

or

$$
\begin{aligned}
{\left[\begin{array}{l}
\dot{x_{1}}(t) \\
\dot{x_{2}}(t)
\end{array}\right]=} & -D\left[\begin{array}{l}
x_{1}(t) \\
x_{2}(t)
\end{array}\right]+A\left[\begin{array}{l}
f_{1}\left(x_{1}(t)\right) \\
f_{2}\left(x_{2}(t)\right)
\end{array}\right]+B\left[\begin{array}{l}
f_{1}\left(x_{1}(t-\tau)\right) \\
f_{2}\left(x_{2}(t-\tau)\right)
\end{array}\right] \\
+C & {\left[\begin{array}{l}
\int_{t-\sigma}^{t} f_{1}\left(x_{1}(\rho)\right) d \rho \\
\int_{t-\sigma}^{t} f_{2}\left(x_{2}(\rho)\right) d \rho
\end{array}\right]+\left[\begin{array}{l}
J_{1}(t) \\
J_{2}(t)
\end{array}\right] }
\end{aligned}
$$

where $f_{i}\left(x_{i}(t)\right)=g_{i}\left(x_{i}(t)\right)=h_{i}\left(x_{i}(t)\right)=\frac{\left|x_{i}(t)+1\right|-\left|x_{i}(t)-1\right|}{2}, i=1,2$.

$$
\begin{gathered}
A=\left(\begin{array}{cc}
\frac{\cos \sqrt{2} t+e^{-t^{2} \sin ^{2} t}}{3} & 0 \\
0 & \frac{\cos t+e^{-t^{2} \sin ^{2} t}}{5}
\end{array}\right) \quad \Longrightarrow \quad\|A\|_{2} \leq \frac{2}{3}, \\
B=\left(\begin{array}{cc}
\frac{\sin \sqrt{2} t+e^{-t^{2} \sin ^{2} t}}{2} & 0 \\
0 & \frac{\cos t+e^{-t^{2} \sin ^{2} t}}{4}
\end{array}\right) \quad \Longrightarrow \quad\|B\|_{2} \leq \frac{1}{2}, \\
C=\left(\begin{array}{cc}
\frac{1}{4} & \frac{1}{2} \\
1 & \frac{1}{5}
\end{array}\right) \quad \Longrightarrow \quad\|C\|_{2}=0.93255, \quad D=\left(\begin{array}{cc}
5 & 0 \\
0 & 4
\end{array}\right) \quad \Longrightarrow \quad\|D\|_{2} \leq 5, \\
J(t)=\left(\begin{array}{c}
\sin \sqrt{2} t+\sin t+e^{-t^{2} \sin ^{2} t} \\
\cos t+\sin \sqrt{5} t
\end{array}\right), \quad \tau=\sigma=3 .
\end{gathered}
$$

It follows that $r<1$, which implies that system (6.1) satisfies all the conditions in Theorem 1. Hence, system (6.1) has exactly one pseudo almost periodic function in the convex set $\mathcal{B}$ (see Figure 1).

\section{Conclusions}

In recent years, there have been extensive results on the problem of the existence and stability of periodic and almost periodic solutions of the chaotic neural networks by numerous authors (see, for example, [6,7,14,15, 16, 18,20,21,23,29]. In this paper, we have studied the global asymptotic stability of the equilibrium point and the existence of pseudo almost periodic solutions for a class of neural networks with mixed delays. We have employed mainly the well known Banach contraction principle as well as the the Brouwer's fixed point Theorem to prove our main results. Finally, an example with its numerical simulations has been provided to demonstrate the feasibility of our results. It should be mentioned that our results are more general and stronger than those in earlier publications which study the periodic and almost periodic solutions. 

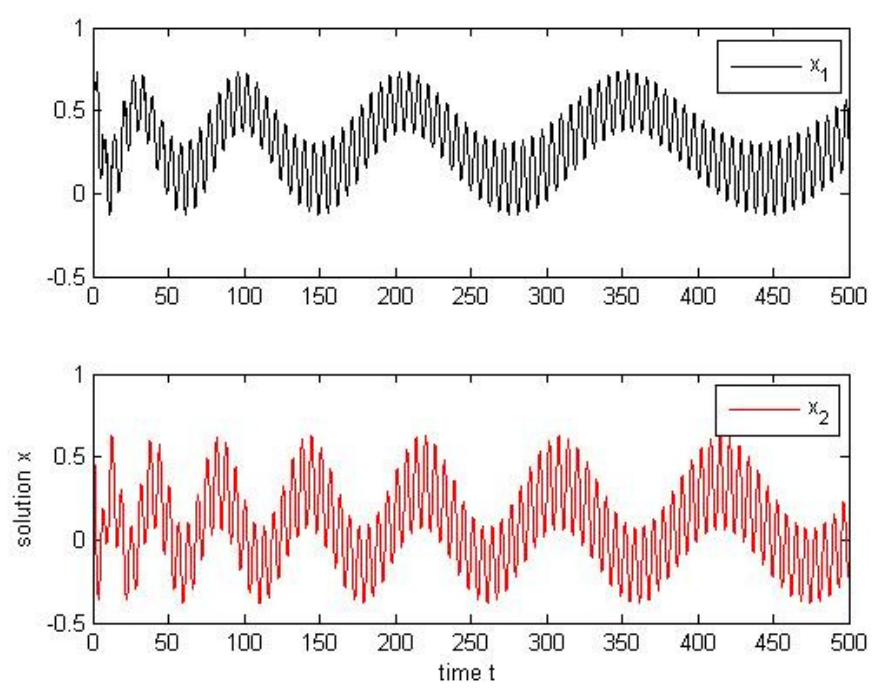

Figure 1. Curve of pap solution of CNNs.

\section{Acknowledgments}

I would like to thank the reviewers and the editor for their valuable suggestions and comments which have led to a much improved paper.

\section{References}

[1] E. Ait Dads, K. Ezzinbi and O. Arino. Pseudo almost periodic solutions for some differential equations in a Banach space. Nonlinear Anal., 28(7):1141-1155, 1997. http://dx.doi.org/10.1016/S0362-546X(97)82865-9.

[2] L. Amerio and G. Prouse. Almost-Periodic Functions and Functional Analysis. Van Nostrand Reinhold Co., New York, 1971.

[3] B. Amir and L. Maniar. Composition of pseudo almost periodic functions and Cauchy problems with operators of non dense domain. Ann. Math. Blaise Pascal, 6(1):1-11, 1999. http://dx.doi.org/10.5802/ambp.110.

[4] B. Ammar, F. Chérif and Adel M. Alimi. Existence and uniqueness of pseudo almost-periodic solutions of recurrent neural networks with time-varying coefficients and mixed delays. IEEE Trans. Neural Networks and Learning Systems, 23(1):109-118, 2012. http://dx.doi.org/10.1109/TNNLS.2011.2178444.

[5] S. Blythe, X. Mao and X. Liao. Stability of stochastic delay neural networks. J. Franklin Institute, 338:481-495, 2001.

http://dx.doi.org/10.1016/S0016-0032(01)00016-3.

[6] J. Cao. New results concerning exponential stability and periodic solutions of delayed cellular neural networks. Phys. Lett. A, 307(2-3):136-147, 2003.

http://dx.doi.org/10.1016/S0375-9601(02)01720-6. 
[7] J. Cao, A. Chen and X. Huang. Almost periodic attraction of delayed neural networks with variable coefficients. Phys. Lett. A, 340:104-120, 2005. http://dx.doi.org/10.1016/j.physleta.2005.04.021.

[8] J. Cao and J. Wang. Global asymptotic stability of a general class of recurrent neural networks with time-varying delays. IEEE Trans. Circuits Syst. I, 50(1):34-44, 2003. http://dx.doi.org/10.1109/TCSI.2002.807494.

[9] F. Chérif. Pseudo almost periodic solutions of a class of chaotic neural network. World Academy of Science, Engineering and Technology, 73, 2011.

[10] F. Chérif. Dynamics and oscillations of GHNNs with time-varying delay. In A.E.P. Villa et al.(Ed.), Artificial Neural Networks and Machine Learning ICANN 2012, volume 7552 of Lecture Notes in Computer Science, pp. 17-24, Berlin Heidelberg, 2012. Springer-Verlag. ISBN 978-3642-33268-5.

[11] F. Chérif. Existence and global exponential stability of pseudo almost periodic solution for SICNNs with mixed delays. J. Appl. Math. Comput., 39(1-2):235251, 2012. http://dx.doi.org/10.1007/s12190-011-0520-1.

[12] T. Diagana. Pseudo almost periodic solutions to some differential equations. Nonlinear Anal., 60(7):1277-1286, 2005.

http://dx.doi.org/10.1016/j.na.2004.11.002.

[13] H.S. Ding, J. Liang, G.M. N'Guérékata and T.J. Xiao. Mild pseudo-almost periodic solutions of nonautonomous semilinear evolution equations. Math. Comput. Modelling, 45(5-6):579-584, 2007.

http://dx.doi.org/10.1016/j.mcm.2006.07.006.

[14] Q. Dong, K. Matsui and X. Haung. Existence and stability of periodic solutions for Hopfield neural network equations with periodic input. Nonlinear Anal., 49(4):471-479, 2002. http://dx.doi.org/10.1016/S0362-546X(01)00113-4.

[15] Z. Gui, W. Ge and X. Yang. Periodic oscillation for a Hopfield neural networks with neutral delays. Phys. Lett. A, 364(3-4):267-273, 2007.

http://dx.doi.org/10.1016/j.physleta.2006.12.013.

[16] S. Guo and L. Huang. Periodic solutions in an inhibitory two-neuron network. J. Comput. Appl. Math, 161(1):217-229, 2003.

http://dx.doi.org/10.1016/j.cam.2003.08.002.

[17] J.K. Hale. Theory of Functions Differential Equations. Springer, New York, 1977.

[18] H. Huang, J. Cao and J. Wang. Global exponential stability and periodic solutions of recurrent cellular neural networks with delays. Phys. Lett. A, 298(56):393-404, 2002. http://dx.doi.org/10.1016/S0375-9601(02)00537-6.

[19] H-X. Li, F.-L. Huang and J.-Y. Li. Composition of pseudo almost-periodic functions and semilinear differential equations. J. Math. Anal. Appl., 255(2):436-446, 2001. http://dx.doi.org/10.1006/jmaa.2000.7225.

[20] B. Liu. Almost periodic solutions for Hopfield neural networks with continuously distributed delays. Math. Comput. Simulation, 73(5):327-335, 2007. http://dx.doi.org/10.1016/j.matcom.2006.05.027.

[21] B. Liu and L. Huang. Existence and global exponential stability of almost periodic solutions for Hopfield neural networks with delays. Neurocomputing, 68:196-207, 2005. http://dx.doi.org/10.1016/j.neucom.2005.05.002. 
[22] B. Liu and L. Huang. Existence and stability of almost periodic solutions for shunting inhibitory cellular neural networks with time-varying delays. Chaos Solitons Fractals, 31(1):211-217, 2007. http://dx.doi.org/10.1016/j.chaos.2005.09.052.

[23] Z. Liu, A. Chen, J. Cao and L. Huang. Existence and global exponential stability of almost periodic solutions of BAM neural networks with continuously distributed delays. Phys. Lett. A, 319(3-4):305-316, 2003. http://dx.doi.org/10.1016/j.physleta.2003.10.020.

[24] L.M. Pecora and T.L. Carroll. Synchronization in chaotic systems. Phys. Rev. Lett., 64(8):821-824, 1990. http://dx.doi.org/10.1103/PhysRevLett.64.821.

[25] V. Singh. Robust stability of cellular neural networks with delay: linear matrix inequality approach. IEEE Proc. Control Theory Appl., 151(15):125-129, 2004.

[26] D.R. Smart. Fixed Point Theorems, volume 66 of Cambridge Tracts in Math. Cambridge University Press, 1980.

[27] Q. Song. Design of controlleron synchronization of chaotic neural networks with mixed time-varying delays. Neurocomputing, 72(7):3288-3295, 2009. http://dx.doi.org/10.1016/j.neucom.2009.02.011.

[28] J. Wang, L. Huang and Z. Guo. Dynamical behavior of delayed Hopfield neural networks with discontinuous activations. Appl. Math. Mod., 33(4):1793-1802, 2009. http://dx.doi.org/10.1016/j.apm.2008.03.023.

[29] B. Xiao. Existence and uniqueness of almost periodic solutions for a class of Hopfield neural networks with neutral delays. Appl. Math. Lett., 22(4):528-533, 2009. http://dx.doi.org/10.1016/j.aml.2008.06.025.

[30] C. Zhang. Pseudo almost periodic functions and their application. PhD thesis, University of Western Ontario, 1992. 